\title{
User Assistance for Intelligent Systems
}

\author{
Stefan Morana $\cdot$ Jella Pfeiffer $\cdot$ Marc T. P. Adam
}

Published online: 3 April 2020

(C) The Author(s) 2020

\section{Introduction}

Intelligent systems have become ubiquitous in modern life and increasingly shift the performance of tasks away from humans (Davenport and Kirby 2016). Although this development has many advantages, the interplay between intelligent systems and humans remains a societal and technological challenge (Maedche et al. 2019; Seeber et al. 2020). Taking humans out of the loop may lead to "mindless" ways of working and cause a range of errors due to unforeseen task complexities. Furthermore, human capabilities cannot always cope with intelligent systems' functionalities (Brynjolfsson and McAfee 2016). In sum, intelligent systems have increased their capabilities and functionalities with a rapid pace and thereby widened the gap to the humans' (cognitive) capabilities to comprehend and utilize these systems.

One way to support humans in the usage of intelligent systems is providing user assistance that can be instantiated

S. Morana $(\bowtie)$

Assistant Professor for Digital Transformation and Information Systems, Saarland University, Campus C3 1,

66123 Saarbruecken, Germany

e-mail: stefan.morana@uni-saarland.de

\section{J. Pfeiffer}

Chair of Digitalisation, E-Business and Operations Management, Justus Liebig University Giessen, Licher Str. 74, 35394 Gießen, Germany

e-mail: jella.pfeiffer@wirtschaft.uni-giessen.de

\section{T. P. Adam}

School of Electrical Engineering and Computing, The University of Newcastle, University Drive, Callaghan, NSW 2308,

Australia

e-mail: marc.adam@newcastle.edu.au in many different forms such as conversational agents, guidance systems, recommendation agents, robo-advisors, and virtual assistants. Many contemporary user assistant systems rely on some form of either speech-based or textbased conversational user interface, both for receiving input from and delivering output to users using natural language processing (Maedche et al. 2019). Recent assistance functionalities in the private life context (e.g., navigation and mobility assistants or smart home assistants) have demonstrated their usefulness. Furthermore, technology giants (e.g., Amazon, Google, and Microsoft) have announced to release even smarter digital assistants to the market. In an organizational context, recent assistance functionalities support users in semi-automatic invoice processing and intelligent calendar management. In light of these increasingly powerful assistance functionalities, the role of user assistance for interactive intelligent systems deserves more research.

Following earlier works by Benyon (2014) and Maedche et al. (2016), we suggest the following definition.

Definition We define user assistance as an intelligent system's capability to assist users while performing their task by means of human-, task-, and/or context-dependent augmentation of the human-computer interaction. User assistance systems bridge the gap between the system's functionalities and the human's individual capabilities with the goal of positively influencing task outcomes.

We classify user assistance for intelligent systems along two dimensions: (1) the degree of interactivity enabled by 

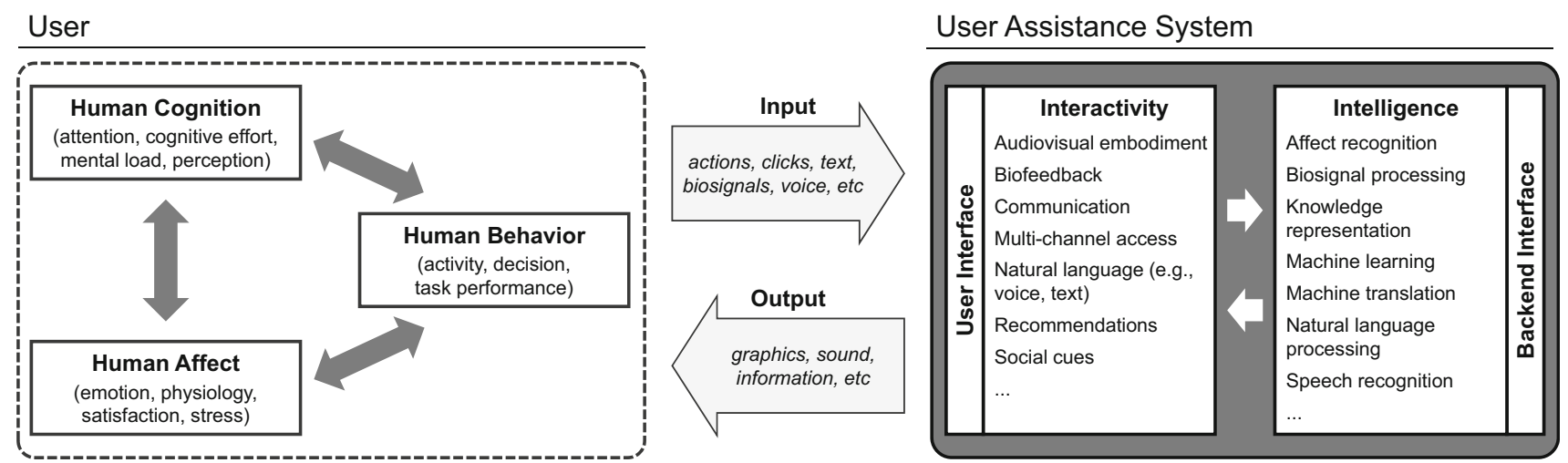

Fig. 1 Conceptualization of a user's interaction with a user assistance system

user assistance, and (2) the degree of intelligence of user assistance. ${ }^{1}$ The degree of interactivity characterizes the assistance system's capability to support humans in an ongoing reciprocal and activating dialog using, potentially, different channels (e.g., text and voice). Highly interactive systems are able to provide feedback in relation to earlier communication points and allow users to control and influence the communication process (Kiousis 2002). The degree of intelligence describes a system's capability to assist the user and is human-, task-, and/or the contextdependent. It covers aspects of artificial intelligence such as the intention to create machines with minds (Haugeland 1989) and emotional intelligence, i.e. the capabilities to manage, understand, use, and perceive emotions (Mayer et al. 2001). Technological advances in machine learning have dramatically increased the capability of systems to recognize human emotion and, based on this, improve their emotional intelligence (Rouast et al. 2019). Other works have shown how assistance systems could learn about the users' context unobtrusively through tracking the users' eyes when using virtual reality equipment (Pfeiffer et al. 2020), automatically sensing the users' mood when interacting with a chatbot (Feine et al. 2019b), or even assist users in training their own emotional intelligence (Astor et al. 2013).

Along the dimensions of interactivity and intelligence, the framework in Fig. 1 provides a simplified conceptualization of a user's interaction with a user assistance system. On the left-hand side, the framework recognizes the importance of considering the user's dynamic interplay of cognition, affect, and behaviour (Jhangiani and Tarry 2014). For instance, user assistance systems are often specifically designed to reduce cognitive effort through

\footnotetext{
${ }^{1}$ User assistance is related to the concept of decision support. However, it needs to be noted that user assistance (1) does not only apply for decision making but virtually any task and (2) focuses specifically on assistance provided in interaction with the user rather than decision support more broadly outside this interaction.
}

targeted recommendations (Jung et al. 2018) or elicit positive emotional states, e.g., satisfaction (Gnewuch et al. 2018). The centre of the framework captures the different input, e.g., eye tracking (Pfeiffer et al. 2020) or text messages (Feine et al. 2019b), and output channels, e.g., avatars graphics (Aljaroodi et al. 2020), between the user and the system (see also Maedche et al. 2019 for a similar differentiation). Finally, on the right-hand side, the framework distinguishes between interactivity and intelligence components of the user assistance system.

One prominent example of user assistance systems that support users in performing IT-based tasks are conversational agents (CA). CAs are "user interfaces that mimic human-to-human communication using natural language processing, machine learning, and/or artificial intelligence" (Schuetzler et al. 2018, p. 94). To increase users' perception of their human-likeness, CAs usually rely on social cues (e.g., profile picture, name, emotional expressions, or delaying the CA's response) to trigger social reactions by their users (Gnewuch et al. 2018; Seeger et al. 2018; Feine et al. 2019a). Their primary mode of communication can be text-based and/or speech-based and used in many different contexts, such as private-life (e.g., entertainment, reminders) and professional contexts (e.g., customer service, employee task support) (Gnewuch et al. 2017). The Nuremberg Institute of Market Decisions (NIM), for example, recently found in a study with 1000 users of conversational agents like Alexa, Google Assistant, and Siri that although 58\% still see several shortcomings in the current versions, $90 \%$ report these assistants to simplify their lives (NIM 2019). Summing up, CAs are a contemporary class of user assistance systems supporting our daily life in many different contexts and situations.

The interview in this special issue with Omer Biran (Conversational AI's Managing Director) and Michael Halfmann (Head of Intelligent Enterprise Center of Excellence) from SAP furthermore shows how large companies already offer CAs to their employees to support 
them in the execution of their tasks (e.g., by directly taking care of simple actions or by directing users to the right page). The interviewees emphasize two key challenges in the design of CAs. First, the "horizontal coverage" principle refers to the challenge of CAs being able to respond to a wide range of possible topics that the user may bring up, without resorting to the infamous "Sorry, I did not understand you" default response. Second, "short exchange" principle refers to the challenge of keeping human-CA conversations brief and creating immediate value for the user (e.g., rapid redirection to appropriate content). This stresses the need to put the purpose and added value of human-agent interactions at the forefront of CA design considerations.

\section{The Special Issue}

The present special issue on User Assistance for Intelligent Systems aims to facilitate research, development, and innovation in user assistance systems. Overall, we received 21 submissions from six different countries, covering a wide range of methods, contexts, and application domains. After a highly competitive and constructive peer review process, three papers about CAs remained. We are delighted to include these three papers in this issue:

In the first paper of this special issue, Diederich et al. (2020) propose and evaluate the design for a CA that offers a human-like interaction experience while mitigating negative effects due to limited responsiveness of the CA. The authors conducted an online experiment to compare users' perceptions of the CA in comparison to an online training system using a conventional graphical user interface without a CA. The research process concluded in the formulation of a design theory, including four design principles. They found that CAs, which follow the proposed design, are perceived as more useful, enjoyable, humanlike, and socially present than an online training system.

In the second paper, Janssen et al. (2020) used a clustering-based approach to develop and evaluate a taxonomy of design elements for domain-specific chatbots. The authors analyzed 103 chatbots from 23 different application domains, based upon which they identified five chatbots archetypes used in practice (i.e. goal-oriented daily, non-goal oriented daily, utility expert, utility facilitator, relationship-oriented). Researchers and practitioners can use the taxonomy to design and classify domain-specific chatbots along the identified dimensions and archetypes.

The third paper by Zschech et al. (2020) presents the design and evaluation of a CA that assists novices in the field of data mining to find the best fitting data mining method for their problem at hand. The CA takes domainspecific problem descriptions in natural language as an input and aims to provide the most suitable class of data mining method as an answer. The authors formulate design principles and features for such text-based CAs and evaluate these empirically using a prototype.

We would like to express our profound gratitude to all the authors as well as the numerous reviewers who have contributed to the research presented in this special issue. A very special thank you goes to the Business and Information Systems Engineering team that made this special issue possible. We hope that researchers and practitioners will find the concepts and scientific results presented in this special issue useful in furthering the field of user assistance for intelligent systems.

Acknowledgements Open Access funding provided by Projekt DEAL.

Open Access This article is licensed under a Creative Commons Attribution 4.0 International License, which permits use, sharing, adaptation, distribution and reproduction in any medium or format, as long as you give appropriate credit to the original author(s) and the source, provide a link to the Creative Commons licence, and indicate if changes were made. The images or other third party material in this article are included in the article's Creative Commons licence, unless indicated otherwise in a credit line to the material. If material is not included in the article's Creative Commons licence and your intended use is not permitted by statutory regulation or exceeds the permitted use, you will need to obtain permission directly from the copyright holder. To view a copy of this licence, visit http://creativecommons. org/licenses/by/4.0/.

\section{References}

Aljaroodi HM, Chiong R, Adam MTP (2020) Exploring the design of avatars for users from Arabian culture through a hybrid approach of deductive and inductive reasoning. Comput Hum Behav 106:106246. https://doi.org/10.1016/j.chb.2020.106246

Astor PJ, Adam MTP, Jerčić P et al (2013) Integrating biosignals into information systems: a NeuroIS tool for improving emotion regulation. J Manag Inf Syst 30:247-278. https://doi.org/10. 2753/MIS0742-1222300309

Benyon D (2014) Designing interactive systems: a comprehensive guide to HCI, UX and interaction design. Pearson Education, London

Brynjolfsson E, McAfee A (2016) The second machine age: work, progress, and prosperity in a time of brilliant technologies. Norton, New York

Davenport TH, Kirby J (2016) Just how smart are smart machines? Sloan Manag Rev 57:20-25

Diederich S, Brendel AB, Kolbe LM (2020) Designing anthropomorphic and communicative enterprise conversational agents. Bus Inf Syst Eng. https://doi.org/10.1007/s12599-020-00639-y

Feine J, Gnewuch U, Morana S, Maedche A (2019a) A taxonomy of social cues for conversational agents. Int J Hum Comput Stud 132:138-161. https://doi.org/10.1016/j.ijhcs.2019.07.009

Feine J, Morana S, Gnewuch U (2019b) Measuring service encounter satisfaction with customer service chatbots using sentiment analysis. In: 14th International conference on Wirtschaftsinformatik, pp 1115-1129 
Gnewuch U, Morana S, Maedche A (2017) Towards designing cooperative and social conversational agents forcustomer service. In: ICIS 2017 proceedings

Gnewuch U, Morana S, Adam MTP, Maedche A (2018) Faster is not always better: understanding the effect of dynamic response delays in human-chatbot interaction. In: Frank (ed) 26th European conference on information systems: beyond digitization - facets of socio-technical change, ECIS 2018, Portsmouth, UK, June 23-28, 2018

Haugeland J (1989) Artificial intelligence: the very idea. MIT Press, Cambridge

Janssen A, Passlick J, Rodríguez Cardona D, Breitner MH (2020) Virtual assistance in any context: a taxonomy of design elements for domain-specific chatbots. Bus Inf Syst Eng. https://doi.org/ 10.1007/s12599-020-00644-1

Jhangiani R, Tarry H (2014) Principles of social psychology - 1st international edition. BCcampus

Jung D, Dorner V, Glaser F, Morana S (2018) Robo-advisory. Bus Inf Syst Eng 60(1):81-86. https://doi.org/10.1007/s12599-018-05219

Kiousis S (2002) Interactivity: a concept explication. New Med Soc 4:355-383. https://doi.org/10.1177/146144480200400303

Maedche A, Morana S, Schacht S et al (2016) Advanced user assistance systems. Bus Inf Syst Eng 58(5):367-370. https://doi. org/10.1007/s12599-016-0444-2

Maedche A, Legner C, Benlian A et al (2019) AI-based digital assistants. Bus Inf Syst Eng 61(4):535-544. https://doi.org/10. 1007/s12599-019-00600-8
Mayer JD, Salovey P, Caruso DR, Sitarenios G (2001) Emotional intelligence as a standard intelligence. Emotion 1:232-242. https://doi.org/10.1037/1528-3542.1.3.232

NIM (2019) Alexa, Google Assistant, Siri und Co: Die digitalen Mitbewohner auf dem Weg zum Mitentscheider und Lebensberater. https://www.nim.org/sites/default/files/medien/359/doku mente/2019_9_nim_pm_smart_speaker_dt-fin.pdf. Accessed 12 Feb 2020

Pfeiffer J, Pfeiffer T, Meißner M, Weiß E (2020) Eye-tracking-based classification of information search behavior using machine learning: evidence from experiments in physical shops and virtual reality shopping environments. Inf Syst Res (in press)

Rouast PV, Adam M, Chiong R (2019) Deep learning for human affect recognition: insights and new developments. IEEE Trans Affect Comput. https://doi.org/10.1109/TAFFC.2018.2890471

Schuetzler RM, Giboney JS, Grimes GM, Nunamaker JF (2018) The influence of conversational agent embodiment and conversational relevance on socially desirable responding. Decis Support Syst 114:94-102. https://doi.org/10.1016/j.dss.2018.08.011

Seeber I, Waizenegger L, Seidel S et al (2020) Collaborating with issues and research opportunities. Internet Res. https://doi.org/ 10.1108/INTR-12-2019-0503

Seeger A-M, Pfeiffer J, Heinzl A (2018) Designing anthropomorphic conversational agents: development and empirical evaluation of a design framework. In: Proceedings of the international conference on information systems, San Francisco

Zschech P, Horn R, Höschele D, Janiesch C, Heinrich K (2020) Intelligent user assistance for automated data mining method selection. Bus Inf Syst Eng. https://doi.org/10.1007/s12599-02000642-3 